\title{
PDZD7 is a modifier of retinal disease and a contributor to digenic Usher syndrome
}

Inga Ebermann, ${ }^{1}$ Jennifer B. Phillips, ${ }^{2}$ Max C. Liebau, ${ }^{3}$ Robert K. Koenekoop, ${ }^{4}$ Bernhard Schermer, ${ }^{3,5}$ Irma Lopez, ${ }^{4}$ Ellen Schäfer, ${ }^{6}$ Anne-Francoise Roux,7,8 Claudia Dafinger, ${ }^{1}$ Antje Bernd, ${ }^{9}$ Eberhart Zrenner, ${ }^{9}$ Mireille Claustres, ${ }^{7,8}$ Bernardo Blanco, ${ }^{2}$ Gudrun Nürnberg, ${ }^{10}$ Peter Nürnberg, ${ }^{5,10}$ Rebecca Ruland, ${ }^{1}$ Monte Westerfield,2 Thomas Benzing, ${ }^{3,5}$ and Hanno J. Bolz ${ }^{1}$

1 Institute of Human Genetics, University Hospital of Cologne, Cologne, Germany. ${ }^{2}$ Institute of Neuroscience, University of Oregon, Eugene, Oregon, USA ${ }^{3}$ Department of Medicine and Centre for Molecular Medicine, University Hospital of Cologne, Cologne, Germany. ${ }^{4}$ McGill Ocular Genetics Laboratory, McGill University Health Centre Research Institute, Montreal, Quebec, Canada. ${ }^{5}$ Cologne Excellence Cluster on Cellular Stress Responses in Aging-Associated Diseases, University of Cologne, Cologne, Germany. ${ }^{6}$ Praxis für Humangenetik, Hamburg, Germany. ${ }^{7} \mathrm{CHU}$ Montpellier, Laboratoire de Génétique Moléculaire, Montpellier, France. ${ }^{8}$ Inserm, U827, Montpellier, France. ${ }^{9} \mathrm{Centre}$ for Ophthalmology, Institute for Ophthalmic Research, University of Tübingen, Tübingen, Germany. ${ }^{10}$ Cologne Center for Genomics and Institute for Genetics, Cologne, Germany.

Usher syndrome is a genetically heterogeneous recessive disease characterized by hearing loss and retinitis pigmentosa (RP). It frequently presents with unexplained, often intrafamilial, variability of the visual phenotype. Although 9 genes have been linked with Usher syndrome, many patients do not have mutations in any of these genes, suggesting that there are still unidentified genes involved in the syndrome. Here, we have determined that mutations in PDZ domain-containing 7 (PDZD7), which encodes a homolog of proteins mutated in Usher syndrome subtype 1C (USH1C) and USH2D, contribute to Usher syndrome. Mutations in PDZD7 were identified only in patients with mutations in other known Usher genes. In a set of sisters, each with a homozygous mutation in USH2A, a frame-shift mutation in PDZD7 was present in the sister with more severe RP and earlier disease onset. Further, heterozygous $P D Z D 7$ mutations were present in patients with truncating mutations in USH2A, G protein-coupled receptor 98 (GPR98; also known as USH2C), and an unidentified locus. We validated the human genotypes using zebrafish, and our findings were consistent with digenic inheritance of $P D Z D 7$ and GPR98, and with PDZD7 as a retinal disease modifier in patients with USH2A. Pdzd7 knockdown produced an Usher-like phenotype in zebrafish, exacerbated retinal cell death in combination with ush2a or gpr98, and reduced Gpr98 localization in the region of the photoreceptor connecting cilium. Our data challenge the view of Usher syndrome as a traditional Mendelian disorder and support the reclassification of Usher syndrome as an oligogenic disease.

\section{Introduction}

Usher syndrome is the leading cause of deafblindness, characterized by congenital deafness and prepubertal onset retinitis pigmentosa (RP) (USH1) or moderate to severe hearing impairment and RP in late adolescence (USH2) (1). Five USH1 and 3 USH2 genes are known. In cochlear hair cells and photoreceptors, Usher proteins interact in a multimolecular complex $(2,3)$. Some Usher proteins share significant similarities: Cadherin-23 (CDH23; USH1D) and Protocadherin-15 (PCDH15; USH1F) belong to the cadherin superfamily, whereas harmonin (Usher syndrome subtype 1C [USH1C]) and whirlin (DFNB31; USH2D) are homologous PDZ proteins that cross-link other Usher proteins.

Many patients with Usher syndrome do not show linkage to mutations in known Usher genes (1), suggesting further genetic heterogeneity. Also, genetic factors that produce the phenotypic variability of Usher syndrome are largely unknown. We hypothesized that mutations in homologs of known Usher proteins may also cause or contribute to the disease. We identified full-length PDZ domain-containing 7 (PDZD7), a homolog of whirlin and harmonin. No biallelic mutations were found in Usher patients. Instead, heterozygous truncating PDZD7 mutations were found

Authorship note: Inga Ebermann, Jennifer B. Phillips, and Max C. Liebau contributed equally to this work.

Conflict of interest: The authors have declared that no conflict of interest exists. Citation for this article: J Clin Invest. 2010;120(6):1812-1823. doi:10.1172/JCI39715 in combination with mutations in USH2A and G protein-coupled receptor 98 (GPR98; also known as USH2C), suggesting a role as either a contributor to digenic disease or a modifier of retinal disease expression. Consistent with a recent report of a homozygous chromosome translocation disrupting PDZD7 in a patient with hearing impairment (4), we demonstrate involvement of $P D Z D 7$ in Usher syndrome. Our histological studies in zebrafish and mouse provide further insight into the molecular role of $P D Z D 7$ in vision and hearing, and functional studies in zebrafish replicated our findings in human families. We therefore suggest that redefining Usher syndrome as an oligogenic trait provides a genetic explanation for its clinical variability.

\section{Results}

PDZD7 encodes a ciliary protein with homology to the USH1C and USH2D proteins. We conducted BLAST searches for homologs of whirlin and harmonin and identified a gene encoding an uncharacterized homolog, PDZD7, on chromosome 10q24.31. $P D Z D 7$ was predicted to encode a protein smaller than harmonin or whirlin with only 2 PDZ domains. We assumed the annotation was incomplete and performed gene prediction with BAC clone AL133215.17. This analysis predicted 16 exons encoding a protein highly similar to harmonin and whirlin (Supplemental Figures 1 and 2; supplemental material available online with this article; doi:10.1172/JCI39715DS1). We verified the predicted coding sequence by PCR on retinal cDNA and identified 

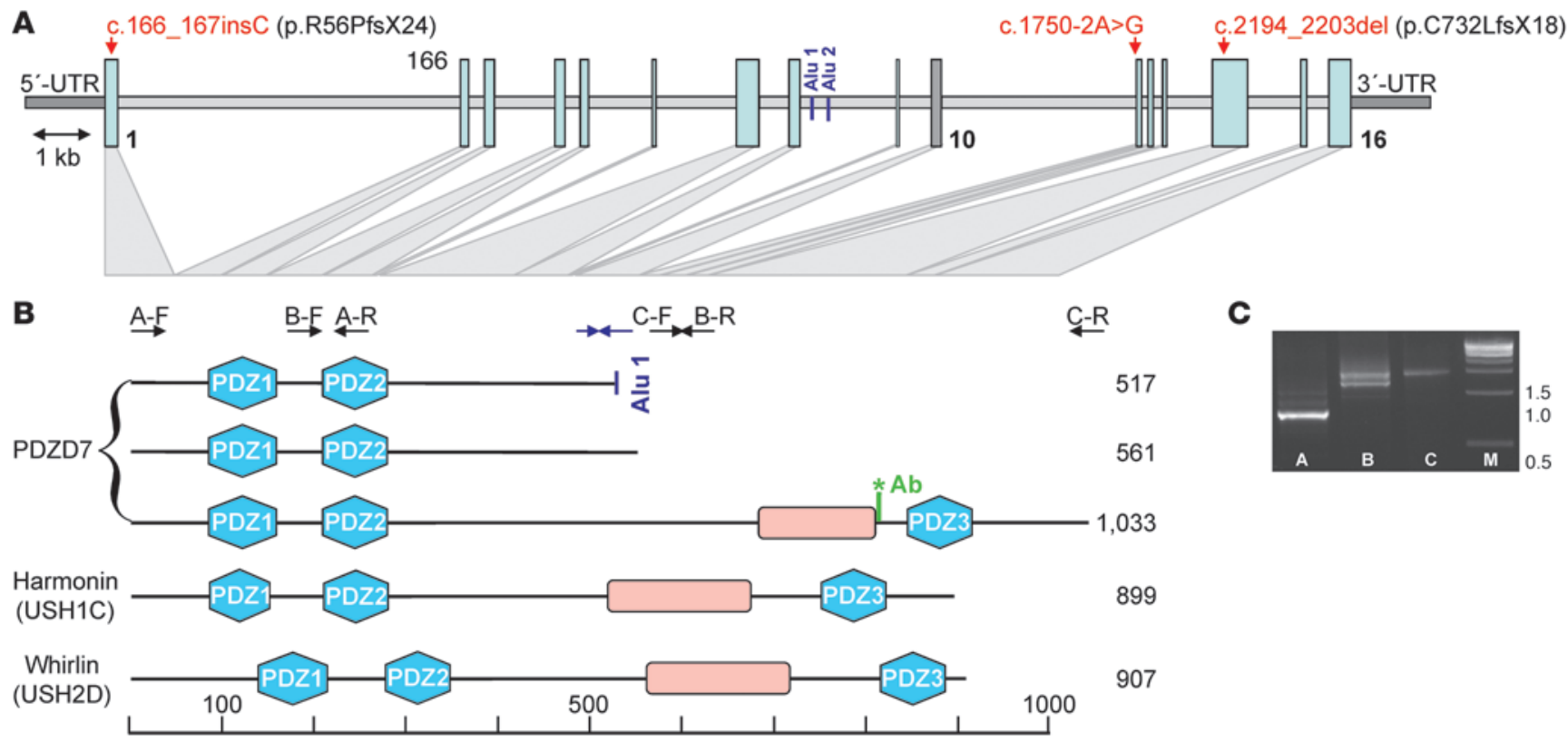

\section{9}
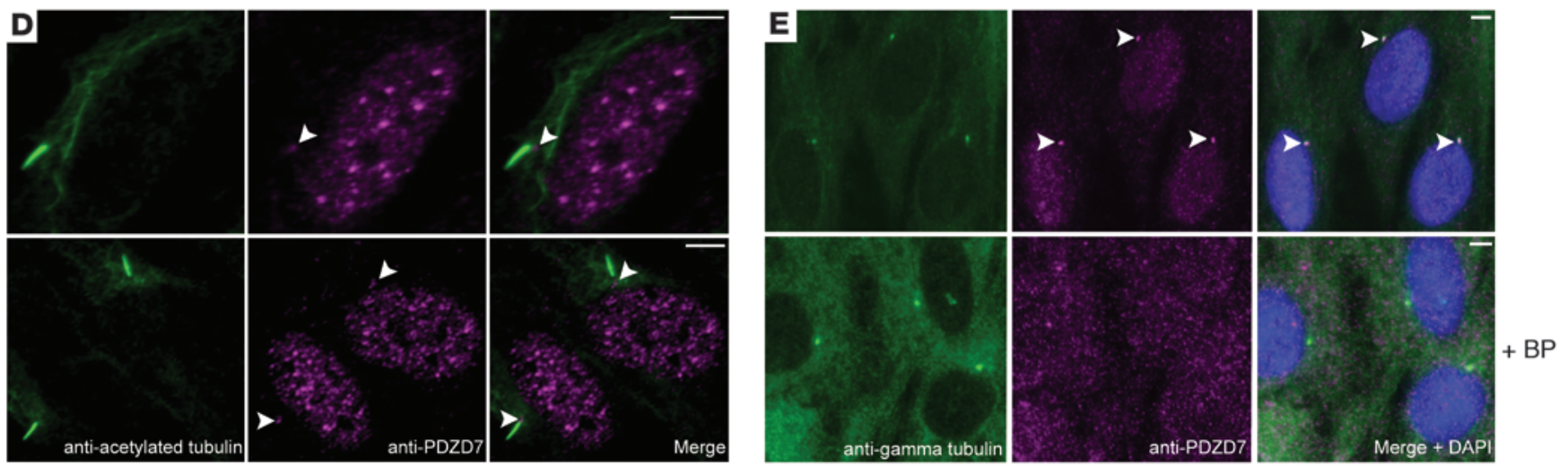

Figure 1

PDZD7 encodes a homolog of harmonin and whirlin localizing to the ciliary base. (A) Genomic structure of PDZD7. Mutations are in red. Grayshaded triangles illustrate relations of exons to protein regions in B. (B) PDZD7 protein isoforms. Intron 8 contains 2 Alu retroelement insertions that modify splicing and produce a short N-terminal transcript (GenBank AK026862) (48). Exons 9 and 10 are alternatively spliced; exclusion from the mature mRNA shifts the reading frame and results in a 561 aa isoform. Ab, Position of epitope for antibody generation. (C) Overlapping PCR amplicons from human retinal cDNA. A, c.1-763 (exon 1 versus exon 5); B, c.512-1899 (exon 3 versus exon 12); C, c.1753-3102+41 (exon 11 versus $3^{\prime}$ UTR); M, 1 kb length standard (Alu-specific amplicon not shown). Arrows indicate primers in relation to the protein in B. The larger band in B represents full-length PDZD7; the smaller one results from exclusion of exons 9 and 10. Blue arrows in B depict primers used to amplify the sequence resulting from Alu-derived splicing that is specific for the first PDZD7 isoform shown (c.1391-Alu in intron 8). The sequences for all primers shown are given in Supplemental Table 2. (D) PDZD7 localizes to the ciliary base. In hTERT-RPE1 cells, costaining with acetylated tubulin revealed PDZD7 localization at the ciliary base and the nucleus. (E) Ciliary base staining was confirmed in costainings of PDZD7 and $\gamma$ tubulin. Preincubation of the PDZD7 antibody with the immunizing peptide abolished this signal. To exclude a remaining weak signal after blocking peptide preincubation, exposure time was prolonged more than 3-fold (lower panel). Arrowheads indicate PDZD7 staining at the ciliary base. Scale bars: $5 \mu \mathrm{m}$.

517,561 , and 1,033 residue isoforms (Figure 1, A-C). PDZD7 is widely expressed (Supplemental Figure 3). To study protein expression, we generated and characterized a polyclonal antibody we believe to be novel (Supplemental Figure 4). Using immunofluorescence studies, we detected the newly predicted C-terminal epitope of PDZD7 at the ciliary base of cultured human retinal pigment epithelial (RPE) cells (Figure 1, D and E) and the $\mathrm{N}$ terminus at the ciliary base in nasal epithelial cells (Supplemental Figure 5). A BLAST query identified the 517 residue isoform in the ciliary proteome database (5).
Evidence for implication of PDZD7 in oligogenic Usher syndrome. We analyzed samples from 188 Usher patients with unknown genetic etiology, but found no PDZD7 alterations. To uncover potential oligogenic genotypes, we screened samples with bi- or monoallelic mutations in known Usher genes. In 4 USH2 families (Figure 2 and Table 1), we found genetic interaction of PDZD7 mutations with Usher alleles, indicating non-Mendelian inheritance in at least 2 of them.

A de novo PDZD7 mutation modifies USH2A disease expression. In a French Canadian USH2 family with a homozygous truncating USH2A mutation (p.C1447QfsX29) in 2 affected sisters, FCa and FCb, we found a 

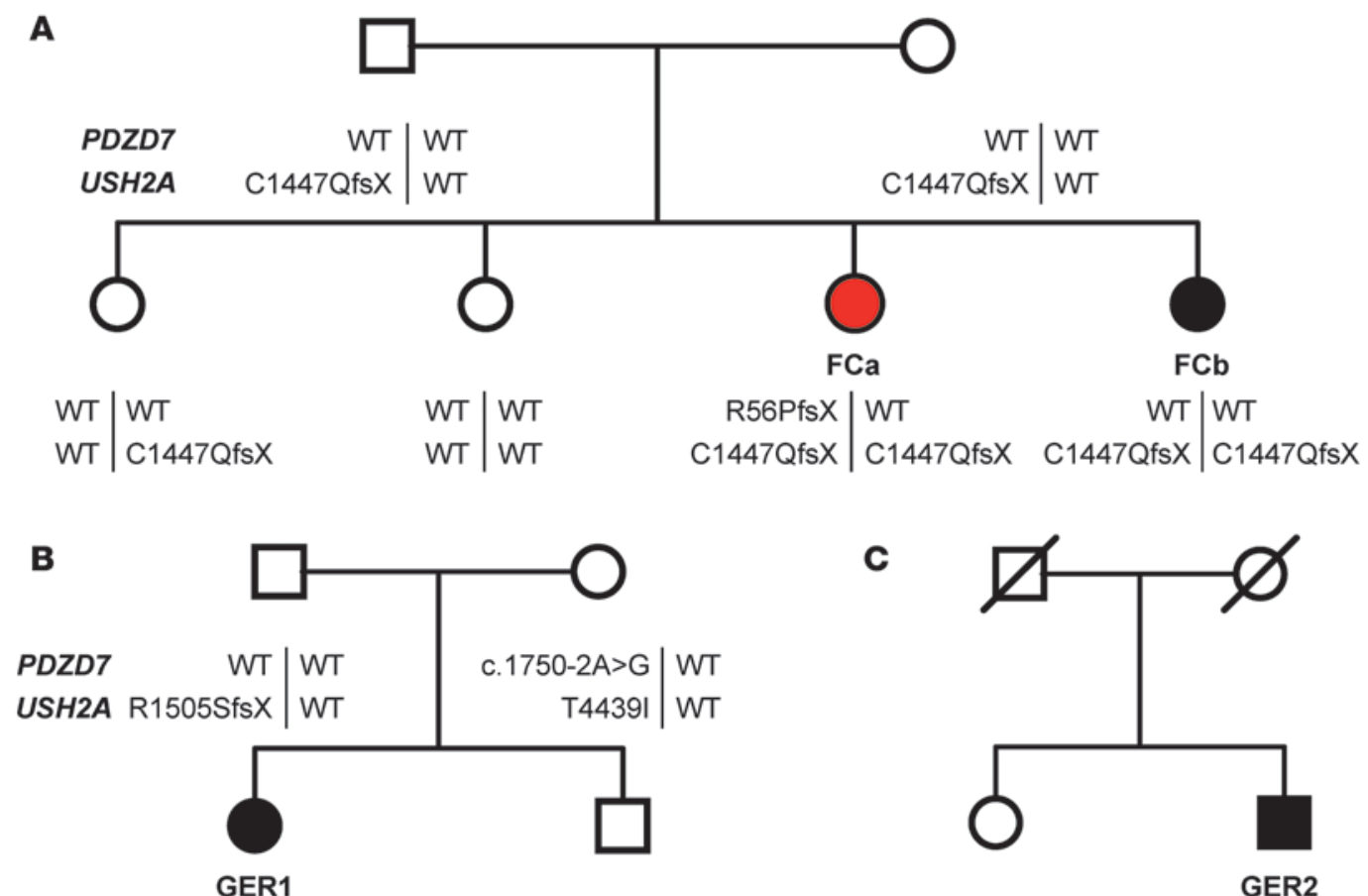

\begin{tabular}{|c|c|c|c|c|c|c|c|c|}
\hline WT & c. $1750-2 A>G$ & WT & WT & PDZD7 & C732LfsX & WT & C732LfsX & WT \\
\hline R1505SfsX & T4439I & R1505SfsX & WT & $\begin{array}{c}\text { GPR98 } \\
\text { (USH2C) }\end{array}$ & WT & WT & A5713LfsX & WT \\
\hline
\end{tabular}

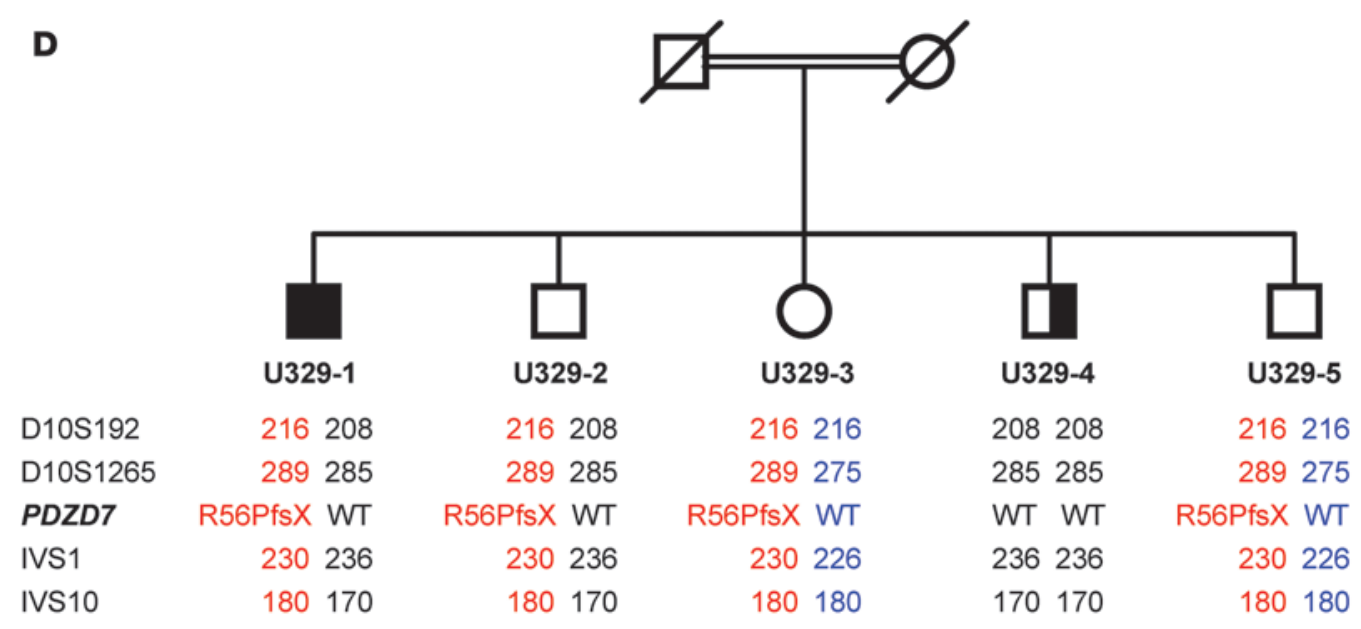

Figure 2

PDZD7 mutations in Usher syndrome type 2 families. (A) Homozygosity for p.C1447QfsX29 ${ }_{\text {uSH2A }}$ in FCa and FCb. The retinal phenotype is more severe in FCa, who carries a de novo PDZD7 mutation, p.R56PfsX24. (B) GER1 is double heterozygous for p.R1505SfsX7uSH2A and a PDZD7 splice site alteration (causing in-frame inclusion of either 16 or 68 unrelated amino acids). The latter may represent a benign variant that does not contribute to the phenotype. (C) Evidence for digenic inheritance due to double heterozygosity for truncating mutations in GPR98 (USH2C), p.A5713LfsX3, and PDZD7, p.C732LfsX18, in GER2. The healthy sister carries the PDZD7 deletion but not the GPR98 mutation. (D) In family U329, 4 siblings carry the PDZD7 mutation p.R56PfsX24 in heterozygous state. Microsatellite marker and sequence analyses largely excluded a second mutation in PDZD7. The index patient, U329-1, carries a heterozygous missense change, p.L3160F, in MYO15A (not shown). Different PDZD7 alleles are indicated by colors. Samples from the parents (first generation cousins) were not available. U329-2 has high frequency hearing loss and a history of long-term working in a noisy environment. U329-4 has asymmetric hearing loss (mild/severe) that is probably unrelated to the hearing impairment in U329-1. IVS1 and IVS10 denote polymorphic CA-repeats (not annotated).

heterozygous frameshift mutation in PDZD7 (p.R56PfsX24) in FCa, the sister with earlier onset and more severe RP (Figure 2A). The insertion occurred de novo in a homonucleotide repeat and was not present in FCb, who displays a much milder retinal affliction (Table 1).
PDZD7 mutation in an USH2A patient with a mild phenotype. GER1, an USH2 patient with a relatively mild phenotype, had heterozygous mutations in USH2A (p.R1505SfsX7) and PDZD7 (exon 11 acceptor splice site, c.1750-2A>G) (Figure 2B). RNA analysis revealed 
aberrant splicing that produces in-frame inclusion of either 16 or 68 unrelated amino acids. GER1 carries another USH2A variation of uncertain pathogenicity, p.T4439I. The healthy mother of GER1 carries p.T4439I $\mathrm{I}_{U S H 2 A}$ and c.1750-2A> $\mathrm{G}_{P D Z D 7}$ in heterozygous state. p.T4439I USH2A $_{\text {has }}$ been previously described in an USH2 patient (6) and was not detected in 463 healthy controls in our study.

Evidence for digenic Usher syndrome involving PDZD7 and GPR98 (USH2C). Our findings in another USH2 patient, GER2, strongly suggest involvement of PDZD7 in digenic USH2. In addition to a heterozygous frameshift mutation, p.A5713LfsX3, in GPR98, we identified a heterozygous truncating PDZD7 mutation, p.C732LfsX18. The healthy sister of GER2 carries the PDZD7 mutation, but not the GPR98 mutation, in heterozygous state (Figure 2C). In GER2, no second mutant allele was detected in GPR98 or PDZD7. Mutation screening was negative for the Usher genes USH2A, DFNB31 (USH2D), and USH3A. Comparative genomic hybridization (CGH) using the Affymetrix 6.0 SNP array and a customized NimbleGen array densely covering the genomic structure of GPR98 did not reveal any gross structural alteration (deletion or duplication) within this gene in GER2 (Supplemental Figures 6 and 7).

A French family, U329, has the same PDZD7 mutation as FCa, p.R56PfsX24, in heterozygous state in 1 Usher patient (U329-1, excluded for USH2A [ref. 7], USH2C, USH2D, and USH3A mutations) and 3 unaffected siblings (Figure 2D). As in FCa, RP in U329-1 is early onset and unusually severe, whereas hearing impairment is typical for USH2. Microsatellite markers excluded monogenic Usher syndrome due to PDZD7, USH2A, or GPR98 mutations. Digenic inheritance involving the Usher genes USH1C, MYO7A (USH1B), USH1G, CDH23 (USH1D), or PCDH15 (USH1F) could not be excluded based on microsatellite marker analysis, but direct sequencing of all coding exons of these genes did not reveal any mutations. Moreover, the microsatellite genotyping results were incompatible with the hypothesis of p.R56PfsX24 modifying the phenotype of a biallelic mutation in a known Usher gene. The results of genomewide SNP genotyping (10K Array) were evaluated for (a) a homozygous disease gene locus that is modified by the PDZD7 mutation and (b) digenic inheritance involving a known autosomal recessive nonsyndromic hearing loss (ARNSHL) locus. Homozygosity mapping did not reveal a candidate gene. In the digenic scenario, the loci comprising the ARNSHL genes TECTA, LHPFL5, COL11A2, TMC1, MYO3A, and MYO15A segregate with the phenotype. We postulated that an ARNSHL gene causing digenic Usher syndrome in conjunction with a PDZD7 mutation would probably meet the following criteria: (a) close functional relationship to and/or (b) physical interaction with a member of the Usher protein complex. To test these possibilities, we examined all coding exons of $M Y O 3 A$ (strong expression in calycal process of retinal photoreceptors; ref. 8) and MYO15A (interacts with the PDZD7 homolog whirlin; ref. 9) by direct sequencing of patient U329-1. Although no sequence alteration was detected in $M Y O 3 A$, a previously described ARNSHL-associated $M Y O 15 A$ missense mutation, p.L3160F (c.9478C>T) (10), was identified in this patient in heterozygous state. This allele was not found in any of the patient's siblings. MYO15A could be amplified from human retinal cDNA only by nested PCR, indicating low abundance in this tissue.

None of the PDZD7 mutations identified in these families were found in healthy control individuals (Table 1). However, 3 in 405 healthy control individuals carried a heterozygous 1-basepair PDZD7 deletion, c.2107delA. Microsatellite marker analysis indicated that these carriers are related and share a PDZD7 haplotype.
PDZD7 interacts with the Usher proteins GPR98 and USH2A. In accordance with our genetic data, we demonstrate that PDZD7 acts as part of the Usher protein complex. A yeast-2-hybrid $(\mathrm{Y} 2 \mathrm{H})$ screen of human fetal brain cDNA (bait: PDZD7 amplicon encoding aa 39-306, including PDZ domains 1 and 2) revealed interaction of PDZD7 (PDZ2 domain) with the C-terminal intracellular domain of GPR98 (approximately 140 residues; isolated as prey 8 times), including its PDZ-binding motif. We confirmed this interaction by coimmunoprecipitation studies and showed that it is mediated by the PDZ2 domain of PDZD7 and the PDZ-binding motif of GPR98. In addition, the first and second PDZ domains of PDZD7 interact with USH2A by coimmunoprecipitation studies (Figure 3). A truncated version of USH2A without the C-terminal PDZ-binding motif showed reduced interaction.

Characterization of the zebrafish orthologs, $p d z d 7 a$ and $p d z d 7 b$. To study the role of PDZD7 in vivo, we characterized pdzd7 in zebrafish, a valuable model for Usher syndrome (11-13), and identified 2 orthologs, $p d z d 7 a$ and $p d z d 7 b$. The exon and protein structures of Pdzd7a and Pdzd7b are highly similar to each other (Figure 4A) and to human PDZD7 (Figure 1A). Pdzd7a is expressed in retinal cells and mechanosensory hair cells of the inner ear (Figure 4, B and D). $P d z d 7 b$ is also expressed in both retinal cells and hair cells, albeit at lower levels than $p d z d 7 a$ (Figure 4, C and E). Because of the higher expression level of $p d z d 7 a$, we concentrated our functional studies on this gene. We obtained a polyclonal antibody (14) raised against the PDZ1 domain of Pdzd7a. With this reagent, we found that the protein is localized in the connecting cilium region of photoreceptors as well as in the apical region of hair cells in the inner ear and neuromasts in young fish (Figure 4, F-H, and Figure 5F). We also noted some nuclear labeling in the inner retina (Figure 4F) that was not predicted by our in situ results, but that is consistent with PDZD7 labeling in cultured cells (Figure 1, D and E). Also notable was the absence of Pdzd7a protein from the photoreceptor synapses at all stages examined. Strong Pdzd7a localization at the ciliary base, as well as more diffuse labeling of photoreceptor outer segments, persisted in adult retinas (Figure 4I), indicating a postdevelopmental function. Due to the high degree of amino acid conservation between zebrafish and mouse, we were able to use our Pdzd7a antibody to label retinas from P10 mice and again found strong labeling at the base of the photoreceptor connecting cilium (Figure 4J).

Simulation of the buman genotypes by knockdown of zebrafish $p d z d 7 a$. We generated a morpholino antisense oligonucleotide to knock down Pdzd7a protein levels transiently by interfering with splicing between exons 1 and 2 (Figure 4A). Using RT-PCR, we identified an aberrant splice form resulting from injection of this morpholino with a truncated, cryptically spliced exon 1 that leads to a shift in the reading frame and an early stop codon. We used the Pdzd7a antibody in Western blots of protein extracts from pools of either uninjected controls or morpholino-injected animals between 3 and 5 days of age (Figure $4 \mathrm{~K}$ ) and found that the protein band of approximately $100 \mathrm{kDa}$, corresponding to the predicted size of Pdzd7a, was greatly diminished in the 3-and 4-day morphants compared with uninjected controls. Protein levels recovered by about $5 \mathrm{dpf}$, as the morpholino effect dissipated. Although we are unable to assay cross-reactivity between this antibody and the $\mathrm{Pdzd} 7 \mathrm{~b}$ protein in vivo, our Western blot analysis did not detect a band of the predicted size of Pdzd7b $(79 \mathrm{kDa})$, indicating that this antibody recognizes Pdzd7a, but not Pdzd7b. Morpholino knockdown of $p d z d 7$ a produced Usherlike symptoms, as previously described for zebrafish Usher gene 


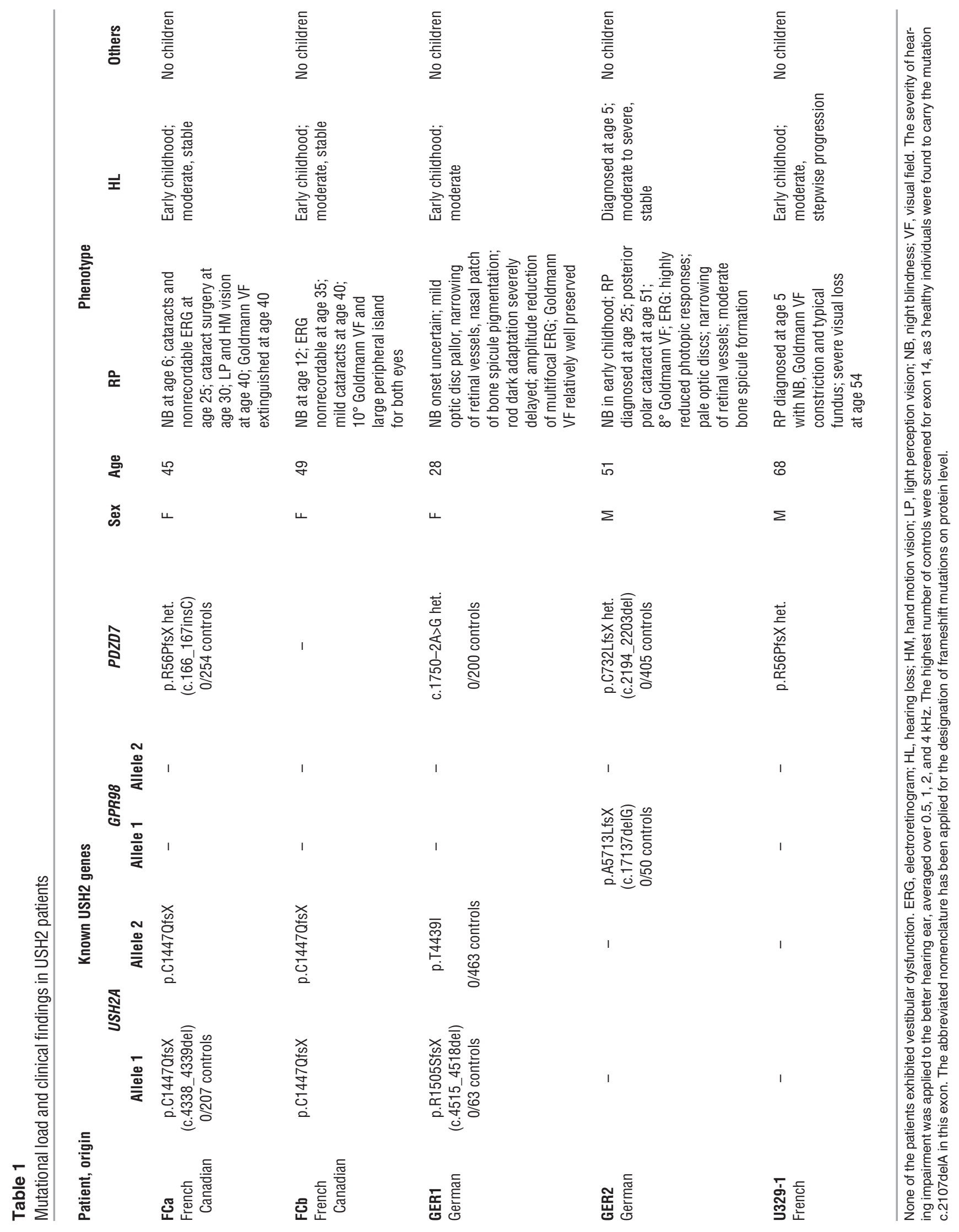


A

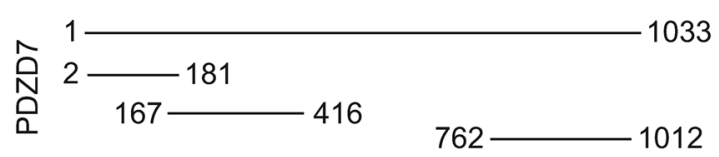

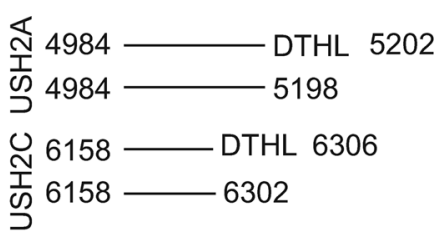

B
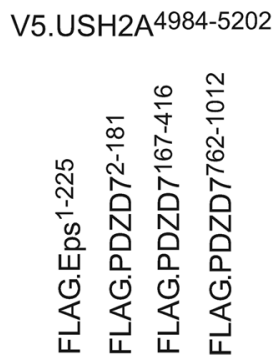

$36 \mathrm{kDa}$
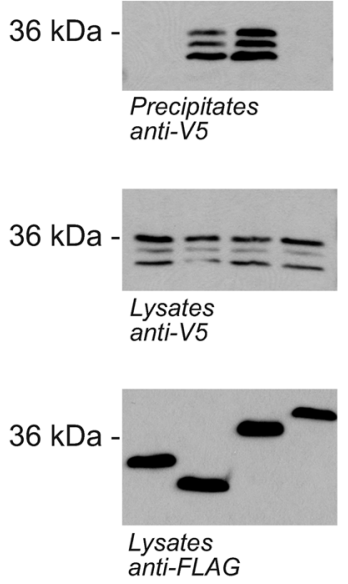

C
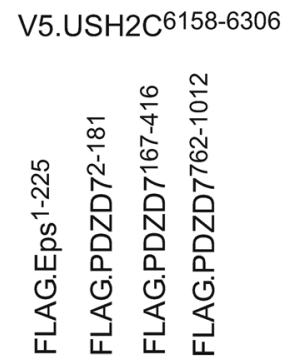

$28 \mathrm{kDa}$

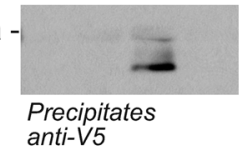

$28 \mathrm{kDa}$
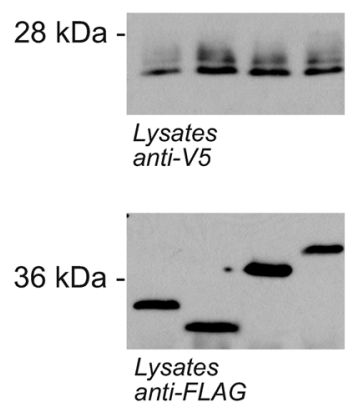

D

\section{V5.PDZD7167-416}
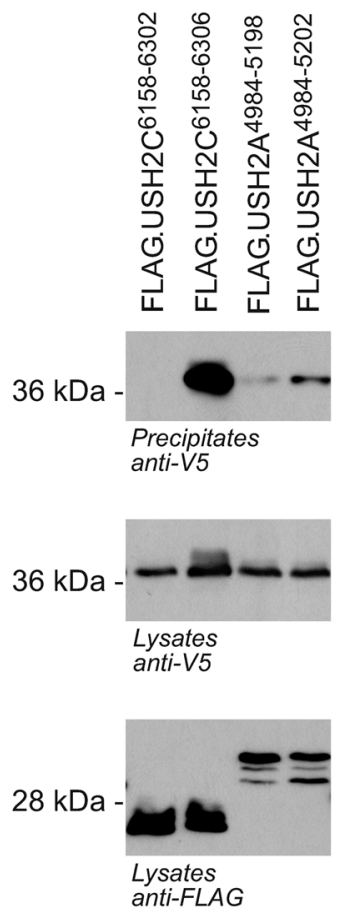

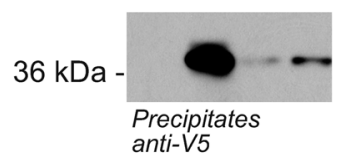

Figure 3

PDZD7 interacts with USH2C (GPR98) and USH2A. (A) Schematic representation of the truncations used for coimmunoprecipitation experiments. (B and C) V5-tagged USH2C ${ }^{6158-6306}$ or USH2A ${ }^{4984-5202}$ was coexpressed with the $3 \mathrm{~N}$-terminally FLAGtagged PDZ domain truncations of PDZD7 or FLAG-tagged Eps ${ }^{1-225}$ as a negative control. Expression of proteins in cellular lysates was confirmed by immunoblotting with V5 and FLAG antibodies, respectively. After immunoprecipitation with FLAG antibody, coprecipitated V5-tagged USH2C ${ }^{6158-6306}$ or USH2A ${ }^{4984-5202}$ was detected with V5-specific antibodies. (D) In the reverse experiment, a V5-tagged PDZD7 truncation coprecipitated with FLAG-tagged USH2C ${ }^{6158-6306}$ or USH2A 4984-5202. Deletion of the PDZ-binding motif (aa DTHL) in the cytoplasmic tails of USH2C and USH2A weakened the interaction with the PDZ2 domain of PDZD7. mutations (11-13), including circling, reduced startle reflex, and disorganized stereocilia (Figure 5, A-D). When we examined the stereocilia of the anterior macula in $p d z d 7$ morphants and controls $(n=3$ in each group, representative examples shown in Figure 5, A-D), we found that $p d z d 7$ a knockdown resulted in abnormal hair bundle morphology. Individual hair bundles in controls $(n=85)$ protruded from the hair cell at an average angle of $74.3^{\circ}$ with an SD of $10.99^{\circ}$, and only 6 of these with an angle of less than $60^{\circ}$. In contrast, hair bundles in the $p d z d 7 a \mathrm{MO}(n=66)$ had an average angle of $37.34^{\circ}$ with an SD of $13.83^{\circ}$, and only 2 of these had an angle greater than $60^{\circ}(P<0.0001$ by 2 -tailed Student's $t$ test).

In the retina, $\mathrm{Pdzd} 7 \mathrm{a}$ protein localization at the connecting cilia was greatly diminished in the knockdown animals (Figure 5G). The inner retina label noted in Figure 4F was undiminished in the knockdown at all stages examined. Because this localization is also inconsistent with our in situ results, we conclude that this label is nonspecific to Pdzd7a.

To determine whether the observed biochemical interactions between PDZD7 and USH2 proteins (Figure 3) function in the zebrafish retina, we assayed localization of Gpr98 and Ush2a using zebrafish-specific antibodies. Both proteins were found at the connecting cilium and at the photoreceptor synapse, consistent with reports in mammals $(15,16)$. After $p d z d 7$ a knockdown, Gpr98 localization was reduced in the connecting cilium region but was unchanged in the synaptic layer (Figure 5, H and I), consistent with the localization of $\mathrm{Pdzd} 7$ a protein. In contrast, Ush2a levels and localization appeared normal in $p d z d 7 a$ knockdown retinas (data not shown).

We explored the potential interactions among Pdzd7a, Usherin, and Gpr98 further by testing behavior and retinal cell death in larvae injected with morpholinos that block ush $2 a$ or $g$ pr 98 function, either alone or in combination with the $p d z d 7 a$ morpholino. Morpholinos injected at half-strength produced no impairment of balance or retinal cell survival when used alone, whereas combined partial pdzd7a;gpr98 knockdown produced Usher-like circling and retinal degeneration (Figure $5 \mathrm{E}$ ). No Usher-like phenotype was observed in combined partial $p d z d 7 a ; u s h 2 a$ knockdown. However, knockdowns of either ush $2 a$ or $p d z d 7 a$ alone produced similar moderate levels of photoreceptor cell death, and this degeneration increased dramatically when embryos were treated with a full dose of ush $2 a$ morpholino combined with a half dose of $p d z d 7 a$ morpholino (Figure 5E; Supplemental Figure 8). The cell death observed in these retinas was restricted to the photoreceptors, and the retinas were otherwise morphologically normal.

\section{Discussion}

Many monogenic disorders, including USH2A (17), present with marked clinical and often intrafamilial variability. The genetic factors underlying this variability are essentially unknown. Here, we show that heterozygosity for a truncating PDZD7 allele, 

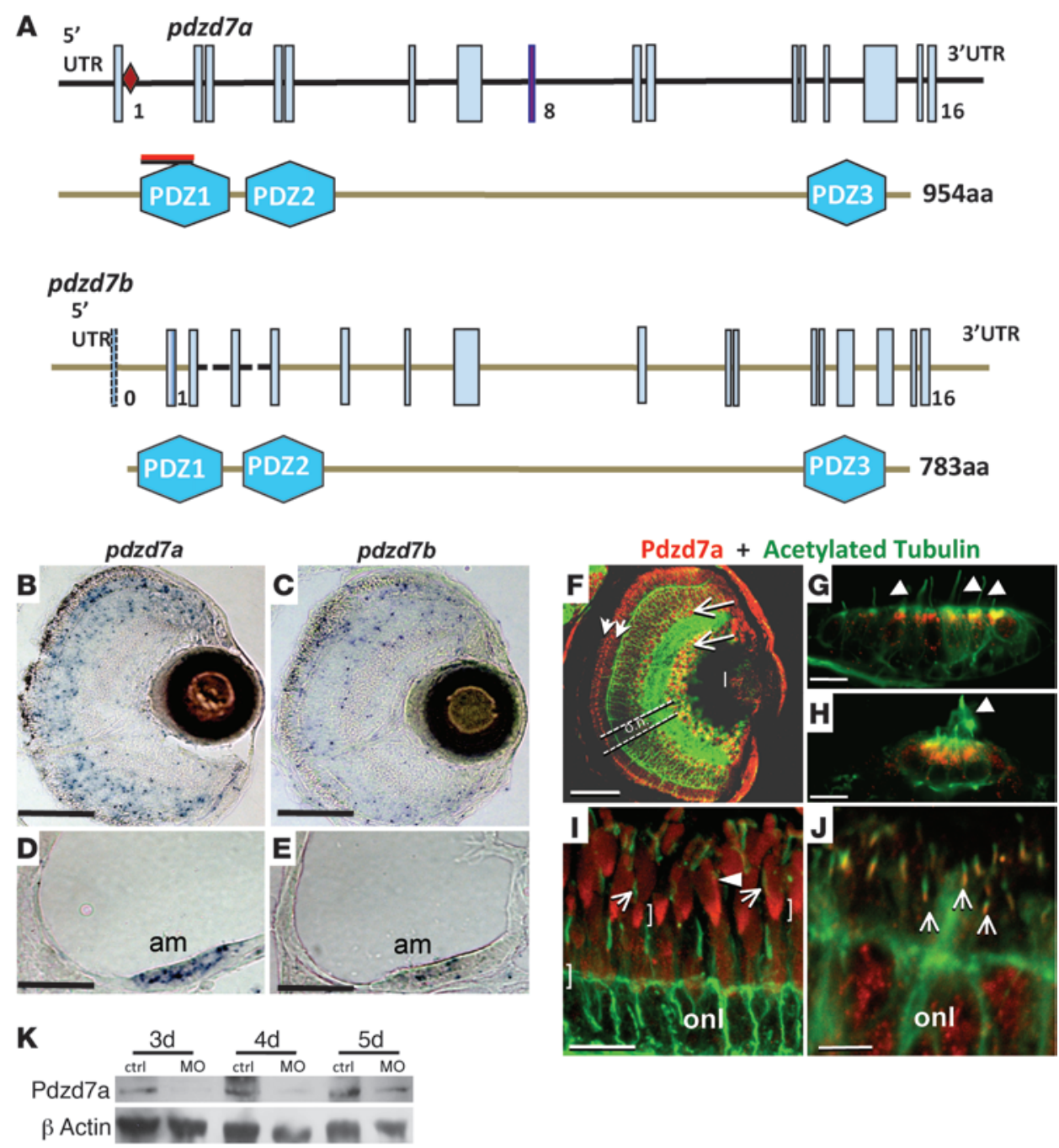

Figure 4

The pdzd7 gene is duplicated in zebrafish and expressed in sensory cells. (A) Genomic structure of $p d z d 7 a$ and $p d z d 7 b$. Exon 8 of $p d z d 7 a$ (purple) is alternatively spliced. The pdzd7a morpholino (red diamond) causes aberrant splicing. The Pdzd7 antibody recognizes 100 aa of the Pdzd7a PDZ1 domain (red bar). (B-E) RNA in situ hybridization. pdzd7a and $p d z d 7 b$ are expressed in the retina $(\mathbf{B}, \mathbf{C})$ and in mechanosensory hair cells (am, anterior macula, shown in $\mathbf{D}, \mathbf{E}$ ). (F-J) Pdzd7a protein localization in sensory cells. (F) At 5dpf, the Pdzd7 antibody (red) labels the region near connecting cilia (short arrows). A subset of cells in the INL and GCL (long arrows) are also labeled. Synaptic layers, neurites, and cilia are visualized with anti-acetylated tubulin (green). Pdzd7a (red) localizes to the apical borders of macular cells $(\mathbf{G})$ beneath the kinocilia (green, arrows) and in ciliated neuromast cells $(\mathbf{H})$. High magnification views of $\mathrm{Pdzd7a}$ (red, brackets) at the base of the connecting cilium (green, arrows) in retinas of adult fish (I) and young mouse $(\mathbf{J})$. Pdzd7a is also observed at lower levels in the outer segments of adult zebrafish photoreceptors (arrowhead in I). on, optic nerve; I, lens; onl, outer nuclear layer. (K) Western blot analysis of $\mathrm{Pdzd7a}$ protein from morpholino and control larvae. $\beta$-actin was used as a loading control. Scale bars: $50 \mu \mathrm{m}$ (B-E); $20 \mu \mathrm{m}$ (F); $10 \mu \mathrm{m}$ (G, H); $20 \mu \mathrm{m}$ (I); $5 \mu \mathrm{m}$ (J).

p.R56PfsX24, aggravates retinal disease in an USH2A family. Intriguingly, the $P D Z D 7$ allele occurred de novo in a sequence motif prone to small insertions or deletions (18). Consistent with PDZD7 acting as a modifier on a sensitized USH $2 \mathrm{~A}^{-/-}$background, partial knockdown of the zebrafish ortholog, $p d z d 7 a$, dramatically enhances photoreceptor cell death in fish with complete knockdown of ush2a. The only previous report of a known genetic basis for intrafamilial discordance in USH involved 2 brothers with a homozygous USH $3 A$ mutation (19). Similar to the PDZD7 mutation we discovered in FCa, an additional heterozygous MYOTA (USH1B) truncation produced a dramatically enhanced phenotype (19). Epistatic interaction was also reported in mice with a hypomorphic Cdh23 (USH1D) allele that modified hearing impairment due to a homozygous Gpr98 mutation (20). In Bardet-Biedl syndrome (BBS), another genetically heterogeneous recessive RP disorder, heterozygous mutations in $B B S 1, B B S 2$, or $B B S 6$ correlate with a more severe phenotype in patients with biallelic mutations at a second BBS locus $(21,22)$.

The unexpected finding of monoallelic PDZD7 mutations in combination with a monoallelic mutation in GPR98 and biallelic mutations in USH2A suggest a functional and possibly physical interaction of the encoded proteins. In support, we show that the 3 proteins indeed interact. As with most Usher proteins, USH2A and GPR98 localize to the photoreceptor connecting cilium region, which plays a pivotal role in trafficking nascent proteins from inner to outer segment $(15,16$, 23). Consistent with our genetic findings and protein interaction data, Pdzd7 is strongly localized in the basal part of the connecting cilium region in zebrafish and at the base of cilia in cultured RPE cells. Notably, Gpr98 localization is reduced in the connecting cilium in $p d z d 7 a$ knockdown zebrafish, but unchanged in the synapse layer where Pdzd7 is not found (Figure 5I). Thus, Pdzd7 apparently localizes Gpr98 in the connecting cilium region, whereas targeting of Gpr98 to the synapse may be mediated by the homologous scaffold proteins, harmonin (15) or whirlin (24). These data provide further support for the inclusion of PDZD7 as a member of the Usher protein complex, consistent with the recent identification of USH1G and harmonin as binding partners (4). PDZD7 is also present in the perinuclear region, a finding that has also been described for its homolog whirlin (25).

Usher-like circling behavior and retinal pathology in combined partial $p d z d 7 a$; $g p r 98$ knockdown in zebrafish (Figure 5E) are consistent with human digenic inheritance of mutations in both genes as 

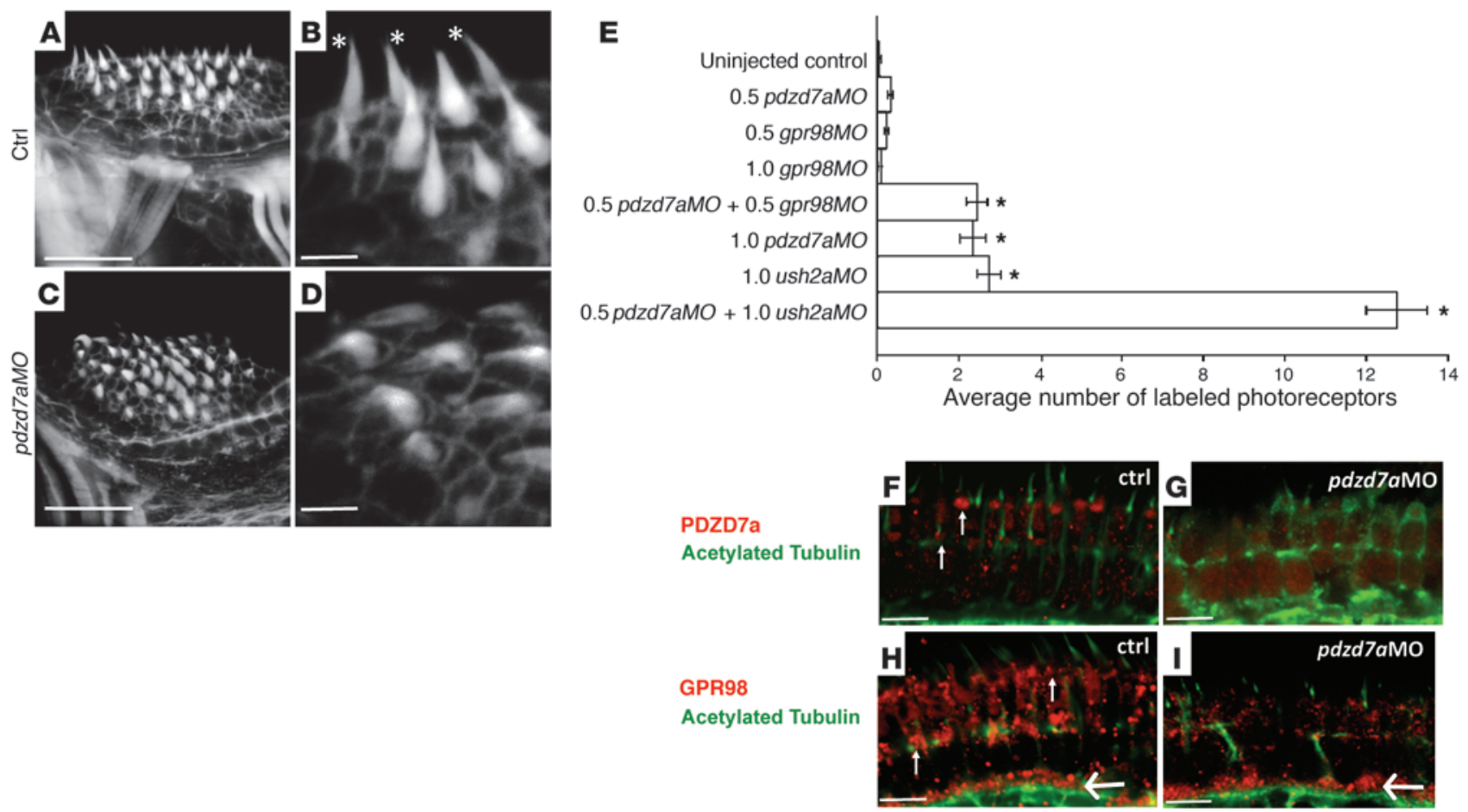

\section{Figure 5}

Loss of Pdzd7a results in structural defects, retinal cell death, and protein mislocalization. (A-D) Phalloidin-stained hair bundles of the anterior crista in $5 \mathrm{dpf}$ control (A, B) and pdzd7aMO-injected (C, D) larvae. Stereocilia in controls are upright and tapered (asterisks), whereas bundles are bent and disorganized after morpholino injection. (E) Graph of average number of dying photoreceptor cells, assayed by anti-active caspase-3 labeling, in control and morpholino-injected fish. Morpholino combinations and dosages (either full: 1.0; or half: 0.5) are indicated for each group. $n=20$ for each category. ${ }^{*} P \leq 10^{-6}$ derived from Student's $t$ test comparing retinal cell death rates in morphant larvae to those of control animals. SEM is indicated by error bars for each group. (F-I) High magnification scans of photoreceptor cells in 5dpf larvae showing localization of Pdzd7 $(\mathbf{F}, \mathbf{G})$ and $\mathrm{Gpr} 98(\mathbf{H}, \mathbf{I})$ antibodies (red) in control and pdzd7aMO injected larvae; acetylated tubulin in green in all panels. In the control, Pdzd7a (red, F) and Gpr98 (red, H) are present at the base of the connecting cilia (small arrows). Images taken from a single focal plane. Gpr98 is also present at the photoreceptor synapse (large arrows), where Pdzd7a is not detected. Labeling by both antibodies is reduced at the connecting cilium after $p d z d 7 a M O$ injection (G, I), whereas synaptic localization is unaffected by $p d z d 7$ knockdown. Scale bars: $10 \mu \mathrm{m}(\mathbf{A}, \mathbf{C}) ; 5 \mu \mathrm{m}(\mathbf{B}$, D); $5 \mu \mathrm{m}$ (F-I).

observed in GER2. Digenism can generally be inferred when (a) the phenotype results from mutations in 2 genes, (b) each gene from such a combination has the potential to cause monogenic disease, and (c) heterozygosity for a mutation in one gene is incapable of causing disease without a simultaneous mutation in the other gene. Although we did not identify patients with biallelic PDZD7 mutations, our knockdown data and the recent findings by Schneider et al. (4) strongly support that condition (b) is also fulfilled.

In patient GER1, however, the contribution of PDZD7 splice site mutation to the phenotype remains inconclusive. Combined partial $p d z d 7 a ; u s h 2 a$ knockdown does not result in an Usher-like phenotype or Ush2a mislocalization in zebrafish. Hence, the p.T4439I $\mathrm{I}_{\text {USH2A }}$ alteration, which affects a highly evolutionary conserved residue that has previously been described in an USH2A patient (6), could be sufficient to cause USH 2 in trans to the truncating USH $2 \mathrm{~A}$ mutation p.R1505SfsX. It may therefore represent a pathogenic mutation whereas c.1750-2A> $\mathrm{G}_{P D Z D 7}$, which produces an in-frame inclusion of unrelated residues, may be a rare benign variant.

The constellation of mutations in patient GER2 suggests genetic noncomplementation of mutations in PDZD7 and GPR98. Because only 1 mutant GPR98 allele has been detected in 3 out of 8 USH2C families reported to date (26-28), PDZD7 is an obvious candidate.
Digenic inheritance has been reported in human RP due to double heterozygosity for recessive mutations in peripherin $(R D S)$ and $R O M 1$, which encode interacting structural components of rod outer segments (29). This situation is similar to the digenic inheritance we report here, involving PDZD7 and GPR98, which encode interacting proteins of the connecting cilium region. Digenic Usher syndrome is probably rare; the only report involves $\mathrm{CDH} 23$ and $\mathrm{PCDH} 15$ mutations in USH1 (30). Digenic inheritance creates challenges for medical geneticists. If mutated genes are located on different chromosomes (as in PDZD7;GPR98), recurrence risk in offspring is $25 \%$. Thus, $P D Z D 7$ mutations should be examined in pedigrees with vertical transmission of combined hearing impairment and RP.

The MYO15A missense change p.L3160F in the second MyTH4 domain identified in U329-1 has previously been described as the causative mutation in a family mapped to the corresponding locus, DFNB3 (10). MYO15A encodes an unconventional myosin that is preferentially expressed in the inner ear and that interacts with the USH2D protein, whirlin. However, the contribution of MYO15A to digenic Usher syndrome in U329-1 is questionable because MYO15A was barely detectable in retinal cDNA. Although an auditory phenotype could result from double heterozygosity for mutations in PDZD7 and MYO15A, additional retinal degeneration 
is unlikely if $M Y O 15 A$ lacks appreciable retinal expression. Moreover, presence of p.L3160F MYO15A $_{\text {in a healthy control has been }}$ reported, and at least one additional deafness gene is predicted for the DFNB3 locus (31). Hence, p.L3160F MYO15A $_{\text {may be a benign }}$ variant, and a heterozygous mutation of an as yet unknown gene with cochlear and retinal function probably contributes to the Usher phenotype of U329-1 (digenic inheritance). Alternatively, p.R56PfsX24 could modify the phenotype of a biallelic mutation in an USH gene. In view of the parental consanguinity, this locus would likely be homozygous in the patient. The recurrent detection of p.R56PfsX24 indicates that the mononucleotide run of 7 cytosines in exon 1 may represent a mutational hot spot and should therefore be screened preferentially for mutations.

Three in 405 healthy control individuals carried a heterozygous 1-basepair deletion in PDZD7, c.2107delA. Microsatellite marker analysis strongly indicated that these carriers are related, consistent with a mutation inherited from a common ancestor. PDZD7 mutations are probably rare and may primarily manifest in combination with alterations of other USH loci.

Our study indicates only partial functional overlap of the homologs PDZD7, harmonin, and whirlin in the retina. Because a heterozygous PDZD7 mutation on a biallelic USH2A background aggravates visual but not auditory impairment, harmonin and whirlin may compensate for PDZD7 haploinsufficiency in cochlear hair cells but not photoreceptors. Consistent with this interpretation, a recent report described a homozygous reciprocal translocation disrupting PDZD7 in a patient with hearing impairment but no retinal degeneration (4). Although retinal and auditory impairment in $p d z d 7 a$ knockdown zebrafish suggest that biallelic human PDZD7 mutations could result in Usher syndrome, it is possible that, analogous to USH1C and DFNB31 mutations $(3,25,32,33)$, the clinical picture depends on the specific isoform(s) affected. The breakpoint in the deafness patient is in intron 10 and should not affect the $\mathrm{N}$-terminal isoforms (Figure $1 \mathrm{~B}$ ). In contrast, homozygous N-terminal truncation of PDZD7, mimicked by the morpholino knockdown, could produce Usher syndrome in humans.

To date, Usher syndrome has been considered a Mendelian (monogenic) disorder. We show that PDZD7 mutations may modify disease severity in triallelic genotypes with 2 mutant alleles in the USH2A gene and that they likely contribute to digenic Usher syndrome. The Usher syndrome families we describe bridge Mendelian and complex inheritance, similar to the hitherto unique pathophysiology of BBS resulting from combined mutations in different ciliary genes. A better understanding of the pathophysiology in Usher syndrome, especially its phenotypic variability, may require reclassifying this disease as an oligogenic disorder. Our study also supports the classification of Usher syndrome as a retinal ciliopathy, similar to BBS, Leber congenital amaurosis, Senior-Loken syndrome, and Joubert syndrome.

\section{Methods}

Patients. The study was approved by the institutional review board of the Ethics Committee, University Hospital of Cologne. Biological samples were donated by the patients after they provided written informed consent in accordance with the Declaration of Helsinki. All patients met the diagnostic criteria for Usher syndrome. 32 USH1, 203 USH2, and 2 USH3 patients of various ethnic origins were investigated. In all USH2 patients, USH2A exon 13 had been sequenced for exclusion of the most prevalent mutation, c.2299delG. 16 USH2 patients were known to carry 2 USH2A mutations (including the discordant sib pair from the FC family).
31 USH2 patients carried heterozygous USH2A mutations (c.2299delG in 26), but mutation screening had not been extended to all USH2A exons. The GRP98 gene (90 exons) had been directly sequenced in 2 USH2 patients, which identified 2 mutation alleles in 1 patient and only 1 mutation allele in patient GER2. Two discordant siblings with biallelic USH3A mutations and 2 siblings with USH2D were also screened for mutations in PDZD7. Direct sequencing of all coding exons for known USH genes was performed for patients FCa and FCb (USH2A), GER1 (USH2A), GER2 (USH2A, GPR98, DFNB31), and U329-1 (USH2A, GPR98, DFNB31, USH3A). Direct sequencing of all coding exons for the ARNSHL genes $M Y O 3 A$ and MYO15A was performed for patient U329-1.

Genetic linkage and mutation analyses. Protocols for analysis of microsatellite markers were described previously (34). Intragenic CA-repeat markers for PDZD7 were amplified with primers: IVS1-CA ( 237 bp), 5'-CTAATCAGCTGGGAAAGCCCTG-3' and 5'-GCTGAGATTTCTTCATAGG-3'; IVS10-CA ( 178 bp), 5'-CCACTGTCCACTGACCAGTGTCTG-3' and 5 '-GCTCTTCATGTGACTCATCACC- 3 '. The 16 coding exons and intronexon boundaries of $P D Z D 7$ were amplified under standard conditions (primers are given in Supplemental Table 1) and directly sequenced using Big Dye version 1.1 and an ABI 377 DNA sequencer (Applied Biosystems). Mutations were verified in ethnically matched control individuals. The 3 healthy carriers of c.2107delA were analyzed for 12 polymorphic microsatellite markers from different loci and for 2 flanking and 2 intragenic markers of the PDZD7 locus. In the U329 family, we performed genomewide linkage analysis using the Affymetrix GeneChip Human Mapping 10K Array Xba142 (version 2.0) (Affymetrix). GRR (35) and PedCheck (36) were used to verify relationships and to identify Mendelian errors. Nonparametric linkage analysis was done with MERLIN (37). Parametric linkage and haplotype analysis was performed by the program ALLEGRO (38). Calculation was done under the hypotheses of (a) autosomal recessive inheritance and (b) autosomal dominant inheritance. In both cases, we assumed full penetrance and disease allele frequency of 0.0001 . Haplotypes were presented graphically with HaploPainter (39). All data handling was performed using the graphical user interface ALOHOMORA (40).

Exclusion oflarge deletions and duplications involving GPR98 in GER2. The Affymetrix Genome-Wide Human SNP Array 6.0 utilizing more than 906,600 SNPs and more than 946,000 probes for the detection of copy number variation was used for the detection of copy number variations. Quantitative data analyses were performed with GTC 3.0.1 (Affymetrix Genotyping Console) using HapMap270 (Affymetrix) as reference file. In addition, to detect and fine map potential deletions and/or duplications within GPR98 and the other 8 known Usher genes, an oligonucleotide microarray CGH analysis was performed. This analysis was carried out with a custom-designed high-resolution $385 \mathrm{~K}$ array (NimbleGen Systems) with oligonucleotide probes (50 mers to 75 mers) densely covering $9.8 \mathrm{Mb}$ of genomic sequence including the genes mentioned above according to the manufacturer's instructions.

Splicing assay for c.1750-2A>G. Reverse transcription from total RNA from whole blood was carried out using RevertAid H Minus M-MuLV Reverse Transcriptase (Fermentas). RT-PCR was carried out with primers located in exon 10 (5'-GTGCTGGCTGTCACCCGCCAC-3') and 14 (5'-CAAGGCAAGTCTCCAGGTAGACG-3'). PCR products were subcloned into CloneJET (Fermentas), and colonies were analyzed by direct sequencing.

Cloning of human PDZD7. A tblastn search with the full-length protein sequences of harmonin and whirlin was performed against the $(\mathrm{nr} / \mathrm{nt})$ database. Gene prediction was based on BAC clone AL133215.17. cDNA primers were designed according to the annotated and predicted coding sequence of PDZD7. To verify the annotated PDZD7 isoform (GenBank AK026862), 1 reverse primer was chosen to amplify the Alu sequence. Primers used to amplify overlapping PCR amplicons for PDZD7 cDNA (Clontech) are provided in Supplemental Table 2. The human retina marathon-ready cDNA kit 
(Clontech) was used for amplification of 5' UTR and 3' UTR. For 5'-RACE, primary amplification was performed using the $5^{\prime}$ UTR primer (5'-GCGGAATCCGAGCCTCATCGCCCATGGG-3') and AP1, followed by semi-nested PCR with AP2 and the $5^{\prime}$ UTR primer. For $3^{\prime}$-RACE, primary amplification was performed using $3^{\prime}$ UTR-1 primer (5'-CGAGCTTGTGGCAGTGGACGGAG-3') and AP1, followed by nested PCR using 3' UTR primer-2 (5'-CCTCCAGACTCCTGATTCTAAGC-3') and AP2. PCR products were subcloned into CloneJET (Fermentas), and colonies were analyzed for PDZD7 sequences.

Expression analysis. RT-PCR was carried out on cDNA from different tissues (retina: reversely transcribed from retinal RNA, Clontech; blood: reverse transcription from total RNA from whole blood; all other tissues: Human Multiple Tissue cDNA Panel I and II, Clontech) with primers located in different exons. Samples were taken after 30 cycles of amplification. Because cDNA from human cochlea was not available, cochlear expression was verified using cDNA from murine cochlea (provided by K. Noben-Trauth, Section on Neurogenetics, National Institute on Deafness and Other Communication Disorders, NIH).

Coimmunoprecipitation experiments. Coimmunoprecipitation experiments were performed as described (41). In brief, HEK293T cells were cultured in DMEM supplemented with $10 \%$ FBS and transiently transfected with the indicated plasmids by the calcium-phosphate method. After incubation for 24 hours, cells were washed and lysed in a $1 \%$ Triton X-100 lysis buffer. After centrifugation $\left(15,000 \mathrm{~g}, 15\right.$ minutes, $\left.4^{\circ} \mathrm{C}\right)$ and ultracentrifugation $(100,000 \mathrm{~g}$, 30 minutes, $\left.4^{\circ} \mathrm{C}\right)$, cell lysates containing equal amounts of total protein were incubated at $4^{\circ} \mathrm{C}$ with anti-FLAG (M2) agarose beads for approximately 1 hour. The beads were washed extensively with lysis buffer, and bound proteins were resolved by $15 \%$ SDS-PAGE and visualized with enhanced chemiluminescence after incubation of the blots with the respective antibodies. Experiments were repeated 3 times with identical results. The following constructs were cloned into pcDNA6-FLAG/9xHis and pcDNA6-V5 for coimmunoprecipitation experiments (target sequences were amplified from human retinal cDNA and verified by direct sequencing): $P D Z D 7$ : amplicons encoding aa 2-181 (comprising PDZ1), aa 167-416 (comprising PDZ2), and aa 762-1012 (comprising PDZ3); USH2A: the intracellular domain of USH2A (WT: aa 4984-5202, $\triangle$ PBM: aa 4984-5198); GPR98: the intracellular domain of GPR98 (WT: aa 6158-6306, $\triangle$ PBM: aa 6158-6302).

Immunofluorescence staining of hTERT-RPE1, HEK293T cells, and human respiratory epithelial cells. hTERT-RPE1 were seeded on coverslips and cultured in DMEM supplemented with 10\% FBS, 2 mM L-glutamine, and $0.348 \%$ sodium-bicarbonate. HEK293T cell culture has previously been described (42). Cilia formation in RPE cells was induced by serum depletion over 48 hours. After washing with ice-cold PBS, cells were fixed. For PDZD7-acetylated tubulin costaining and the PDZD7-FLAG costaining, we used 4\% PFA, $\mathrm{pH} 7.5$, and $0.05 \%$ Triton X-100 for 8 minutes at room temperature. For PDZD7- $\gamma$ tubulin costaining, cells were fixed for 8 minutes in ice-cold methanol. Cells were washed 3 times with PBS, and double immunofluorescence staining was performed sequentially with the antibodies as indicated. The 6-11-1b acetylated tubulin and the $\gamma$ tubulin antibody were obtained from Sigma-Aldrich. After washing, slides were incubated with the secondary antibody, Cy2- or Alexa Fluor 488-labeled anti-mouse, and Cy3-labeled anti-rabbit IgG, washed again, mounted in a commercially available antifade kit (Invitrogen), and subjected to immunofluorescence microscopy with a microscope (Axiovert200) equipped with the ApoTome System and a CCD camera (Jena; all were obtained from Carl Zeiss MicroImaging Inc.). The appropriate controls were performed without the first and/or second primary antibodies. Human respiratory epithelial cells were obtained by transnasal brush biopsy using a cell collector (Cytobrush Plus; Medscand Malmö) and were suspended in RPMI 1640 without supplements. Samples were spread onto glass slides. Cells were fixed with $4 \%$ PFA in PBS for 5 minutes and were permeabilized with $0.2 \%$ Triton X-100 in PBS for 10 min- utes before blocking with $5 \%$ normal donkey serum and $1 \%$ BSA at room temperature over 90 minutes. Cells were incubated with primary antibodies for 60 minutes (VHL, Santa Cruz Biotechnology Inc.; PDZD7, Abnova), washed, and incubated with secondary antibodies for 20 minutes.

Human PDZD7 antibody. The following peptide sequence was chosen for rabbit immunization: GRYHKPRKARPPLPR (residues 810-824). Blast analysis against the human proteome database was performed to exclude crossreactivity with other proteins. Antibody generation and affinity purification against each peptide were performed by Eurogentec (Seraing). For antibody characterization, HEK293T cells were cotransfected with FLAG.Podocin and either FLAG.PDZD7 ${ }^{314-503}$ or FLAG.PDZD7762-1012 . The FLAG.Podocin construct, HEK293T cell culture, and the transfection methods have previously been described $(42,43)$ and were used to exclude unspecific binding of the antibody either to the FLAG tag or to any overexpressed protein.

Y2H screening. The GAL4-based $\mathrm{Y} 2 \mathrm{H}$ system was used for the identification of interaction partners. The bait (PDZD7 amplicon encoding aa 39-306, including PDZ domains 1 and 2) was subcloned into PGBT9 (Clontech). $\mathrm{Y} 2 \mathrm{H}$ screening of a human fetal brain cDNA library was performed at the Deutsches Krebsforschungszentrum (DKFZ), as described (44).

Zebrafish $p d z d 7$ orthologs. A tblastx search was performed with the human $P D Z D 7$ sequence against the Danio rerio nr database. A homologous gene, $p d z d 7 a$, was identified on zebrafish chromosome 13 . The region from the $5^{\prime}$ UTR to the end of exon 5 is covered by clone CR93236, and exons 6-16 are on clone BX936291. pdzd7b was identified on chromosome 12 and is predicted by GENSCAN 00000031688 in that region. cDNA primers were designed according to the annotated and predicted coding sequences, and the coding sequences were amplified from cDNA of $6 \mathrm{dpf}$ larvae using REDTaq ReadyMix PCR Reaction Mix (Sigma-Aldrich) under standard conditions. Initiation ATGs were verified using a forward primer located in the putative $5^{\prime}$ UTR $100 \mathrm{bp}$ upstream of the initiation ATG. The termination codons were verified using reverse primers in the predicted $3^{\prime}$ UTR. Gaps in the gene sequence of the zebrafish assembly in the region of $p d z d 7 b$ were sequenced using flanking primers.

Zebrafish strains. Procedures with zebrafish were approved by the University of Oregon IACUC. All experiments were performed in the Oregon $\mathrm{AB}$ background. Larval and adult fish were staged and maintained as described (45). Some embryos were treated with 1-phenyl 2-thiourea (Sigma-Aldrich) to block melanization.

Morpholino injections. Morpholino oligonucleotides were obtained from GeneTools. The $p d z d 7 a$ GT sequence targeting the splice donor site of exon 1 is $5^{\prime}$-CTTGAGACTCACCATCGACAGGCGC-3'. The $u s h 2 a$ GT sequence targeting the splice donor site of exon 6 is $5^{\prime}$-GTACGACCTTATGCTTACCTGTTGG- $3^{\prime}$. The gpr 98 GT sequence targeting the splice donor site of exon 22 is $5^{\prime}$-AAGTTGATCCTGTTACCTCAGATAG-3'. All morpholinos were titrated for efficacy and injected into 1- or 2-cell stage embryos at concentrations of between 6 and 46 ng. For each morpholino, a full dose was defined as the lowest concentration at which a swimming phenotype was observed. For partial knockdown morpholino treatments (denoted as 0.5 in the text), full effective dose concentrations (denoted as 1.0) were halved and injected either alone or in combination with another morpholino. Total RNA was extracted from pooled samples of morpholino-injected larvae and uninjected controls ( $n \geq 30$ for each group) using the Qiagen RNeasy Kit. Reverse transcription reactions were performed using random hexamers and Superscript Reverse Transcriptase III (Invitrogen). Alternative splicing was detected by sequencing fragments amplified by PCR using template cDNA from morpholino-treated larvae with uninjected controls. cDNA sequences were analyzed using Lasergene software (DNASTAR).

Western blot of zebrafish protein. Embryos were homogenized in $10 \mathrm{mM}$ Tris-HCl, pH 7.4, 150 mM NaCl, 1 mM EDTA, 0.1\% Triton X-100, 0.1\% $\mathrm{SDS}$, and $5 \mathrm{mg} / \mathrm{ml}$ protease inhibitor cocktail (Pierce). Samples were run 
on an $8 \%$ acrylamide gel and transferred to nitrocellulose membrane with Nupage reagents (Invitrogen). Membranes were washed in PBS $2 \times 15$ minutes, incubated in blocking solution ( $3 \%$ NFDM in PBS) for 1 hour at room temperature, then in primary antibody (diluted 1:1000) plus 1:5000 mouse anti-actin in blocking solution overnight at $4^{\circ} \mathrm{C}$. Membranes were rinsed $3 \times 5$ minutes in PBS, then incubated in donkey anti-rabbit-HRP (Jackson Laboratory) for 1 hour at room temperature. Membranes were rinsed $3 \times 5$ minutes in PBS. HRP was detected using the ECL Plus Detection System (Pierce). To detect actin, membranes were rehydrated and incubated in donkey anti-mouse-HRP for 1 hour, rinsed $3 \times 5$ minutes in PBS, and labeled with the ECL Plus Detection System (Pierce).

In situ bybridization and immunohistochemistry. More than 20 eyes from each experimental and control group were analyzed in all zebrafish retinal histological assays. In some experiments, fish were raised in $0.003 \%$ phenylthiourea (PTU) to inhibit pigment formation. $5 \mathrm{dpf}$ larvae were fixed in $4 \%$ PFA in PBS overnight at $4^{\circ} \mathrm{C}$. After 2 washes in a solution of PBS containing $0.01 \%$ Tween- 20 , tissues were dehydrated in methanol at $-20^{\circ} \mathrm{C}$ overnight, rehydrated in descending concentrations of methanol in PBST, washed 4 times in PBST containing 10\% sucrose, and cryoprotected in 30\% sucrose in PBS overnight at $4^{\circ} \mathrm{C}$. Tissues were then embedded in agarose blocks and sunk in $30 \%$ sucrose overnight. Blocks were rapidly frozen in liquid $\mathrm{N} 2$; $16-\mu \mathrm{m}$ sections were cut at $-20^{\circ} \mathrm{C}$, mounted on slides coated with PolyL-Lysine Solution (Sigma-Aldrich), and air-dried for a minimum of 2 hours at room temperature. Slides were either stored at $-20^{\circ} \mathrm{C}$ or used immediately. Whole eyes were obtained from euthanized $\mathrm{AB}$ adult zebrafish and $\mathrm{P} 10$ mice of mixed genetic backgrounds and processed as described above. ISH was performed as described (46) with digoxigenin-labeled probes against pdzd7a nucleotides 684-1830 and pdzd7b nucleotides 960-1769. For antibody staining, sections were ringed with a hydrophobic marker and blocked in a solution of $10 \%$ NGS with $2 \%$ BSA in PBS for at least 1 hour at room temperature. Primary antibodies were diluted in block solution and incubated overnight at $4^{\circ} \mathrm{C}$. Antibodies and working concentrations were as follows: rabbit antibodies against the PDZ1 domain of zebrafish Pdzd7 (aa 99-198) and the C-terminal PDZ-binding domains of Gpr98 and Usherin (aa 5132-5231) were commercially generated by genetic immunization (14) and used at a dilution of 1:1000; mouse anti-acetylated tubulin (Sigma-Aldrich) was at 1:500; rabbit anti-active caspase-3 (BD Pharmingen) was at 1:500. After washing 5 times in PBS, sections were incubated with secondary antibody (Alexa Fluor 488 anti-mouse or Alexa Fluor 568 anti-rabbit; Invitrogen) diluted 1:200 in block solution in the dark for 2 hours at room temperature. Sections were then washed 5 times in PBS and mounted in Vectashield (Vector Laboratories). For retinal apoptosis analysis, we examined cryosectioned morpholino-injected $5 \mathrm{dpf}$ larvae stained with anti-active caspase- 3 antibody and counted labeled cells in serial sections of the morphant retinas (47). For whole-mount imaging of stereocilia, $5 \mathrm{dpf}$ larvae were fixed in BT (4\% PFA, $0.15 \mathrm{mM} \mathrm{CaCl}_{2}, 4 \%$ sucrose in PBS) and then permeabilized with 5\% Triton X-100 3-4 hours at room temperature to dissolve otoliths. Larvae were rinsed 4 times in PBS, then incubated in $2.5 \mu \mathrm{M}$ Alexa Fluor 488-phalloidin (Molecular Probes) overnight at $4{ }^{\circ} \mathrm{C}$, and rinsed in PBS. 10 ears were analyzed in both experimental and control groups. Images were captured on a Zeiss LSM5 Confocal or Bio-Rad Radiance 2100 Confocal.

URLs. BLAST searches were conducted by NCBI BLAST analysis (http://blast.ncbi.nlm.nih.gov/Blast.cgi). The complete PDZD7 gene was predicted using the GENSCAN gene prediction server (http://genes. mit.edu/GENSCAN.html). PDZD7 protein domain analysis was carried out with ScanProsite (http://www.expasy.org/tools/scanprosite). Protein alignment of PDZD7, whirlin, and harmonin was performed using DIALIGN (http://bibiserv.techfak.uni-bielefeld.de/dialign) and BOXSHADE 3.21 (http://www.ch.embnet.org/software/BOX_form.html).

Accession codes. The sequences of full-length homo sapiens PDZD7 (access FJ617449), zebrafish $p d z d 7 a$ (access FJ624206), and $p d z d 7 b$ (access FJ624207) have been deposited in GenBank.

Statistics. In all zebrafish assays, statistical comparisons were performed between control and morphant zebrafish using a 2-tailed Student's $t$ test. $P$ values equal to or less than 0.001 were considered significant.

\section{Acknowledgments}

We are indebted to the families who have participated in this study. We thank Michaela Thoenes, Jeremy Wegner, and Lise Larrieu for technical assistance. This work was supported by the Deutsche Forschungsgemeinschaft (BO2954/1-2, BE2212, and SCHE1572 to H.J. Bolz, T. Benzing, and B. Schermer), by Forschung contra Blindheit: Initiative Usher-Syndrom e.V., the Gertrud Kusen-Stiftung (to H.J. Bolz), NIH grants DC04186 and HD22486 (to M. Westerfield), and an American Heart Association fellowship to J.B. Phillips.

Received for publication May 1, 2009, and accepted in revised form February 24, 2010.

Address correspondence to: Hanno J. Bolz, Bioscientia Center for Human Genetics, Konrad-Adenauer-Str. 17, 55218 Ingelheim, Germany. Phone: 49.6132.781.206; Fax: 49.6132.781.298; E-mail: hanno.bolz@bioscientia.de.

H.J. Bolz's present affiliations are: Bioscientia Center for Human Genetics (Ingelheim, Germany) and Institute of Human Genetics, University Hospital of Cologne (Cologne, Germany).
1. Cohen M, Bitner-Glindzicz M, Luxon L. The changing face of Usher syndrome: clinical implications. Int J Audiol. 2007;46(2):82-93.

2. Kremer H, van Wijk E, Marker T, Wolfrum U, Roepman R. Usher syndrome: molecular links of pathogenesis, proteins and pathways. Hum Mol Genet. 2006;15 Spec No 2:R262-R270.

3. Ebermann I, et al. A novel gene for Usher syndrome type 2: mutations in the long isoform of whirlin are associated with retinitis pigmentosa and sensorineural hearing loss. Hum Genet. 2007;121(2):203-211.

4. Schneider E, et al. Homozygous disruption of PDZD7 by reciprocal translocation in a consanguineous family: a new member of the Usher syndrome protein interactome causing congenital hearing impairment. Hum Mol Genet. 2008;18(4):655-666.

5. Gherman A, Davis EE, Katsanis N. The ciliary proteome database: an integrated community resource for the genetic and functional dissection of cilia. Nat Genet. 2006;38(9):961-962.
6. Dreyer B, et al. Spectrum of USH2A mutations in Scandinavian patients with Usher syndrome type II. Hum Mutat. 2008;29(3):451.

7. Baux D, et al. Molecular and in silico analyses of the full-length isoform of usherin identify new pathogenic alleles in Usher type II patients. Hum Mutat. 2007;28(8):781-789.

8. Dose AC, Hillman DW, Wong C, Sohlberg L, LinJones J, Burnside B. Myo3A, one of two class III myosin genes expressed in vertebrate retina, is localized to the calycal processes of rod and cone photoreceptors and is expressed in the sacculus. Mol Biol Cell. 2003;14(3):1058-1073.

9. Delprat B, et al. Myosin XVa and whirlin, two deafness gene products required for hair bundle growth, are located at the stereocilia tips and interact directly. Hum Mol Genet. 2005;14(3):401-410.

10. Nal N, et al. Mutational spectrum of MYO15A: the large $\mathrm{N}$-terminal extension of myosin XVA is required for hearing. Hum Mutat. 2007;28(10):1014-1019.
11. Ernest S, Rauch GJ, Haffter P, Geisler R, Petit C, Nicolson T. Mariner is defective in myosin VIIA: a zebrafish model for human hereditary deafness. Hum Mol Genet. 2000;9(14):2189-2196.

12. Seiler C, et al. Duplicated genes with split functions: independent roles of protocadherin 15 orthologues in zebrafish hearing and vision. Development. 2005;132(3):615-623.

13. Sollner C, et al. Mutations in cadherin 23 affect tip links in zebrafish sensory hair cells. Nature. 2004;428(6986):955-959.

14. Chambers RS, Johnston SA. High-level generation of polyclonal antibodies by genetic immunization. Nat Biotechnol. 2003;21(9):1088-1092.

15. Reiners J, Nagel-Wolfrum K, Jurgens K, Marker T, Wolfrum U. Molecular basis of human Usher syndrome: deciphering the meshes of the Usher protein network provides insights into the pathomechanisms of the Usher disease. Exp Eye Res. 2006; 83(1):97-119. 
16. Liu X, et al. Usherin is required for maintenance of retinal photoreceptors and normal development of cochlear hair cells. Proc Natl Acad Sci US A. 2007;104(11):4413-4418.

17. Schwartz SB, et al. Disease expression in Usher syndrome caused by VLGR1 gene mutation (USH2C) and comparison with USH2A phenotype. Invest Ophthalmol Vis Sci. 2005;46(2):734-743.

18. Rodenhiser DI, Andrews JD, Mancini DN, Jung JH, Singh SM. Homonucleotide tracts, short repeats and $\mathrm{CPG} / \mathrm{CPNpG}$ motifs are frequent sites for heterogeneous mutations in the neurofibromatosis type 1 (NF1) tumour-suppressor gene. Mutat Res. 1997;373(2):185-195.

19. Adato A, Kalinski H, Weil D, Chaib H, Korostishevsky M, Bonne-Tamir B. Possible interaction between USH1B and USH3 gene products as implied by apparent digenic deafness inheritance. Am J Hum Genet. 1999;65(1):261-265.

20. Johnson KR, Zheng QY, Noben-Trauth K. Strain background effects and genetic modifiers of hearing in mice. Brain Res. 2006;1091(1):79-88.

21. Katsanis N, et al. Triallelic inheritance in BardetBiedl syndrome, a Mendelian recessive disorder. Science. 2001;293(5538):2256-2259.

22. Badano JL, et al. Heterozygous mutations in BBS1, BBS2 and BBS6 have a potential epistatic effect on Bardet-Biedl patients with two mutations at a second BBS locus. Hum Mol Genet. 2003;12(14):1651-1659.

23. Jacobson $S G$, et al. Usher syndromes due to MYO7A, PCDH15, USH2A or GPR98 mutations share retinal disease mechanism. Hum Mol Genet. 2008;17(15):2405-2415.

24. van Wijk E, et al. The DFNB31 gene product whirlin connects to the Usher protein network in the cochlea and retina by direct association with USH2A and VLGR1. Hum Mol Genet. 2006; 15(5):751-765.

25. Mburu P, et al. Defects in whirlin, a PDZ domain molecule involved in stereocilia elongation, cause deafness in the whirler mouse and families with DFNB31. Nat Genet. 2003;34(4):421-428.
26. Ebermann I, et al. GPR98 mutations cause Usher syndrome type 2 in males. J Med Genet. 2009; 46(4):277-280.

27. Hilgert $\mathrm{N}$, et al. A large deletion in GPR98 causes type IIC Usher syndrome in male and female members of an Iranian family. J Med Genet. 2009; 46(4):272-276.

28. Weston MD, Luijendijk MW, Humphrey KD, Moller C, Kimberling WJ. Mutations in the VLGR1 gene implicate G-protein signaling in the pathogenesis of Usher syndrome type II. Am J Hum Genet. 2004;74(2):357-366.

29. Kajiwara K, Berson EL, Dryja TP. Digenic retinitis pigmentosa due to mutations at the unlinked peripherin/RDS and ROM1 loci. Science. 1994;264(5165):1604-1608.

30. Zheng QY, et al. Digenic inheritance of deafness caused by mutations in genes encoding cadherin 23 and protocadherin 15 in mice and humans. Hum Mol Genet. 2005;14(1):103-111.

31. Belguith $\mathrm{H}$, et al. Screening of the DFNB3 locus: identification of three novel mutations of MYO15A associated with hearing loss and further suggestion for two distinctive genes on this locus. Genet Test Mol Biomarkers. 2009;13(1):147-151.

32. Ahmed ZM, et al. Nonsyndromic recessive deafness DFNB18 and Usher syndrome type IC are allelic mutations of USHIC. Hum Genet. 2002;110(6):527-531.

33. Verpy E, et al. A defect in harmonin, a PDZ domaincontaining protein expressed in the inner ear sensory hair cells, underlies Usher syndrome type 1C. Nat Genet. 2000;26(1):51-55

34. Ebermann I, Lopez I, Bitner-Glindzicz M, Brown C, Koenekoop RK, Bolz HJ. Deafblindness in French Canadians from Quebec: a predominant founder mutation in the USH1C gene provides the first genetic link with the Acadian population. Genome Biol. 2007;8(4):R47.

35. Abecasis GR, Cherny SS, Cookson WO, Cardon LR. GRR: graphical representation of relationship errors. Bioinformatics. 2001;17(8):742-743.

36. O'Connell JR, Weeks DE. PedCheck: a program for identification of genotype incompatibilities in linkage analysis. Am J Hum Genet. 1998;63(1):259-266.

37. Abecasis GR, Cherny SS, Cookson WO, Cardon LR. Merlin--rapid analysis of dense genetic maps using sparse gene flow trees. Nat Genet. 2002;30(1):97-101.

38. Gudbjartsson DF, Jonasson K, Frigge ML, Kong A. Allegro, a new computer program for multipoint linkage analysis. Nat Genet. 2000;25(1):12-13.

39. Thiele H, Nurnberg P. HaploPainter: a tool for drawing pedigrees with complex haplotypes. Bioinformatics. 2005;21(8):1730-1732.

40. Ruschendorf F, Nurnberg P. ALOHOMORA: a tool for linkage analysis using $10 \mathrm{~K}$ SNP array data. Bioinformatics. 2005;21(9):2123-2125.

41. Benzing T, Gerke P, Hopker K, Hildebrandt F, Kim E, Walz G. Nephrocystin interacts with Pyk2, p130(Cas), and tensin and triggers phosphorylation of Pyk2. Proc Natl Acad Sci U S A. 2001;98(17):9784-9789.

42. Huber TB, et al. Podocin and MEC-2 bind cholesterol to regulate the activity of associated ion channels. Proc Natl Acad Sci U S A. 2006;103(46):17079-17086.

43. Huber TB, Kottgen M, Schilling B, Walz G, Benzing $\mathrm{T}$. Interaction with podocin facilitates nephrin signaling. J Biol Chem. 2001;276(45):41543-41546.

44. Albers M, et al. Automated yeast two-hybrid screening for nuclear receptor-interacting proteins. $\mathrm{Mol}$ Cell Proteomics. 2005;4(2):205-213.

45. Westerfield M. The zebrafish book. A guide for the laboratory use of zebrafish (Danio rerio). 5 th ed. Eugene, OR: University of Oregon Press; 2007.

46. Jensen AM, Walker C, Westerfield M. mosaic eyes: a zebrafish gene required in pigmented epithelium for apical localization of retinal cell division and lamination. Development. 2001;128(1):95-105.

47. Phillips J, Chiem JL, Westerfield M. Zebrafish Usher genes are necessary for retinal cell function and survival. ARVO abstracts, \#4494. 2007.

48. Sela N, Mersch B, Gal-Mark N, Lev-Maor G, HotzWagenblatt A, Ast G. Comparative analysis of transposed element insertion within human and mouse genomes reveals Alu's unique role in shaping the human transcriptome. Genome Biol. 2007;8(6):R127. 